\title{
Sign Communication for People with Disabilities Using Kinect Technology at Home
}

\author{
Juan J. Ojeda-Castelo \\ Applied Computing Group \\ University of Almeria \\ Almeria, Spain
}

Luis Iribarne-Martinez

Applied Computing Group, Dept. of Computer Science

University of Almeria

Almeria, Spain

\author{
Cesar Bernal-Bravo \\ Dept. of Education \\ University of Almeria \\ Almeria, Spain
}

\begin{abstract}
Communication with other people and their environment, is an essential skill for people with special needs. In this study we present a communicator which interprets a series of commands by means of corporal expressions. These body expressions are learned by a gesture recognition system according to the requirements and disability of the user. Each of the commands adapt themselves to daily tasks. The system learn gestures and associates them with concrete actions that the user wants to do or needs at that moment.
\end{abstract}

\section{Introduction}

Some people with disabilities may need an augmentative and alternative communication because they have difficulties with speech. People who need an alternative language because they are not able to pronounce words but they understand verbal language and people who need an augmented language because they do not understand so well speech and they make sentences without coherence [4]. There are two kinds of AAC systems depending on the individual characteristics, aided approaches and unaided approaches. The main difference of these systems is whether they requires some form of equipment or not. [2].

People who suffer some physical or intellectual disability can present diverse problems to communicate verbally. The Kinect sensor is characterized by a thick mobility where it identifies the movements that do the biggest muscular groups of the body (legs, arms, etc), while other devices as Intel Real Sense and Leap Motion are characterized by a thin mobility where it is possible to identify more precise movements, like that they are capable of doing the hands or the face. The gestures recognition as Sushmita [3] has been incorporated by it into a big variety of applications of different nature, as they can be: Recognition of the language of signs, navigation or manipulation in virtual environments, communication in video conferences and so on.

Between the software that is included to realize an alternative and augmentative communication, which is completely necessary for the users who can have difficulty of expressing themselves with other people are the communicators. Some examples of the communicators who are used to today are: PiktoPlus 2.0: [5], Scout: [7], e-Mintza: [6]. These communicators have the main features that are developed for mobile devices and use pictographs. This communicator (Figure 1) has the purpose that the user can make certain requests with gestures by pictographs. In addition, the user can choose if you want to make the gestures with his right arm or left arm (see Figure 1a). The Figure $1 \mathrm{~b}$ you can see as you can choose between different foods and the final screen shows (Figure 1c) request of food that the user has chosen. This communicator (Figure 1) has the purpose that the user can make certain requests with gestures by pictographs. In addition, the user can choose if you want to make the gestures with his right arm or left arm (see Figure 1a). The Figure 1b you can see as you can choose between different foods and the final screen shows (Figure 1c) request of food that the user has chosen. This communicator has the advantage with respect to communicators described above that the user only needs to do movements with his body to interact with it and as a consequence it can be used by a greater number of people. However, the other communicators user need touching the screen and if for instance they have a deformity in their hands they can not use the application developed for tablet but it has no problem with the one presented in this paper.

\section{Architecture}

The main idea about the operation of the system is that every gesture has associated an action. The system of recognition will consist of a number of states, which two of them will be statics: the initial state and the final state. The initial state will identify when the process of recognition of a certain gesture begins and the final state in order to know when the recognition of the gesture finishes and checks that gesture has been carried out to execute the corresponding action. 


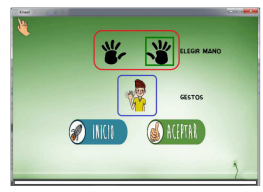

(a)

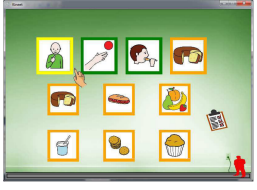

(b)

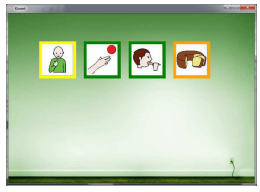

(c)

Figure 1. Screens of communicator.

\begin{tabular}{lccc}
\hline Gesture & Adult man & Adult woman & Kid \\
\hline Me & 0.9 & 0.7 & 0.4 \\
Wish & 0.5 & 0.5 & 0.2 \\
Eat & 0.3 & 0.2 & 0.1 \\
Drink & 0.8 & 0.9 & 0.5 \\
Listen to music & 0.7 & 0.5 & 0.3 \\
Play & 1 & 0.8 & 0.6 \\
Rest & 0.4 & 0.2 & 0.1 \\
Have a shower & 0.3 & 0.4 & 0.1 \\
TABLE 1. ACCURACY RATE OF GESTURE RECOGNITION
\end{tabular}

The other intermediate states will establish the accuracy of the gesture, how many more states will define more precise will be the gesture you store, however, the system will limit the number of states for which the performance was not very expensive. There will be a joint assigned as absolute and another that will be relative. The joint will be the relative to remain in motion during the realization of the gesture, while the absolute will remain static.

Once successfully recognized the gesture, the system will communicate the module action of the gesture that has been recognized to perform the appropriate action in the environment where you are. Some examples of gestures recognized are shown in Figure 2.

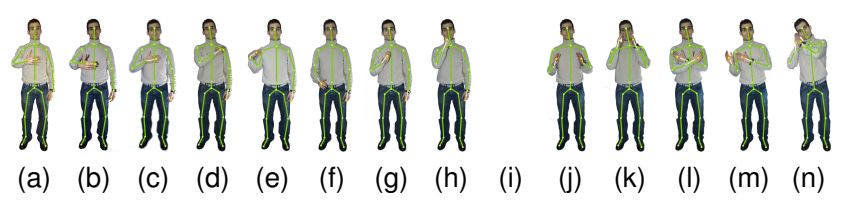

Figure 2. Gestures Assigned in the System.

\section{Results}

The tests have been carried out with different user profiles in response to different physical characteristics of the same as are the height, length of limbs and physical disability. As can be seen in the table 1, have submitted the hit rate that each have one of the gestures in the implemented system, identifying each of them with an action in the home environment. In this way we can observe that the hit rate of the gesture associated with the light has a very high value in comparison with other gestures such as assigned to the heating. All the associated actions have the possibility to upload or lower its intensity through the gestures go up and go down.

\section{Conclusion and Future Work}

The gesture recognition has had more success in the adult individuals that in children, having gestures that have been easier to recognize than others. This result has been due to the fact that some gestures has a very similar pattern recognition, this similarity causes the system does not know that gesture exactly has been recognized and may cause a high error rate, identifying the opposite gesture. In successive phases of the project is to perform a algorithm of gesture recognition that is more accurate using artificial neural networks and in addition, the system will continue an automatic process with respect to the recognition of gestures.

\section{Acknowledgments}

This project has been supported by the Ministry of Education (TIN2013-41576-R). This work was funded by the EU ERDF and the Spanish Ministry of Economy and Competitiveness (MINECO) under Projects TIN2013-41576-R and the Andalusian Regional Government (Spain) under Project P10-TIC-6114. This work also received funding from the CEiA3 and CEIMAR consortiums.

\section{References}

[1] Braun, A., Dutz, T.,\& Kamieth, F. (2013, May). Capacitive sensorbased hand gesture recognition in ambient intelligence scenarios. In Proceedings of the 6th International Conference on PErvasive Technologies Related to Assistive Environments (p. 5). ACM.

[2] Gevarter, C., OReilly, M. F., Rojeski, L., Sammarco, N., Lang, R., Lancioni, G. E., \& Sigafoos, J. (2013). Comparisons of intervention components within augmentative and alternative communication systems for individuals with developmental disabilities: A review of the literature. Research in developmental disabilities, 34(12), 4404-4414.

[3] Mitra, S., \& Acharya, T. (2007). Gesture recognition: A survey. Systems, Man, and Cybernetics, Part C: Applications and Reviews, IEEE Transactions on, 37(3), 311-324.

[4] Simion, E. (2014). Augmentative and Alternative CommunicationSupport for People with Severe Speech Disorders. Procedia-Social and Behavioral Sciences, 128, 77-81.

[5] Piktoplus 2.0 - Accessed 13-06-2015 - http://piktoplus.com/

[6] e-Mintza - Accessed 13-06-2015 - http://fundacionorange.es/ fundacionorange/en/project/emintza.html

[7] Sc@ut - Accessed 13-06-2015 - Sc@ut http://scaut.ugr.es/scaut/ 